\title{
TRATAMENTO FARMACOLÓGICO DA COVID-rg À LUZ DAS EVIDÊNCIAS: OS PRINCIPAIS FÁRMACOS, SUAS INDICAÇÕES E SEU MANEJO TERAPÊUTICO, UMA REVISÃO INTEGRATIVA
}

\author{
PHARMACOLOGICAL TREATMENT OF COVID-I9 IN THE LIGHT OF EVIDENCE: THE \\ MAIN DRUGS, THEIR INDICATIONS AND THEIR THERAPEUTIC MANAGEMENT, AN \\ INTEGRATIVE REVIEW
}

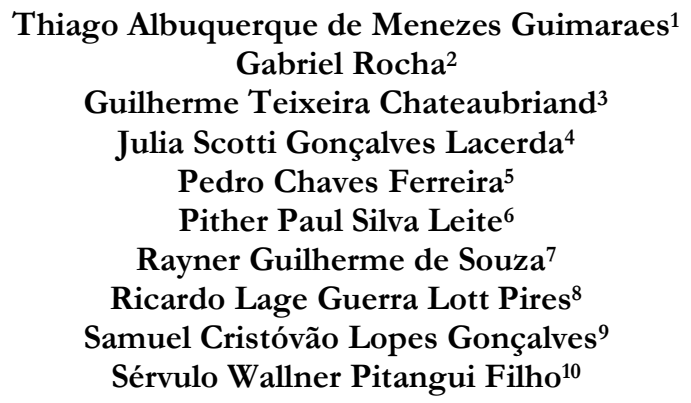

RESUMO: Introdução: A Pandemia de COVID is trouxe, em curto espaço de tempo, um grande número de pacientes infectados com cerca de $20 \%$ deles evoluindo para a forma severa ou crítica da doença, com necessidade de internação hospitalar (WHO, 202I). A grande busca por serviços de saúde e o elevado número de mortes trouxe pressa à ciência para o descobrimento de possíveis medicamentos e ações terapêuticas que mitigassem as consequências danosas da pandemia. Objetivos: Assim, o presente estudo se presta a discutir, à luz das melhores evidências e recomendações, os principais fármacos analisados no tratamento da doença (dexametasona, tocilizumabe, remdesivir, imunoglobulina, ivermectina e hidroxicloroquina, entre outros), suas indicações (ou contraindicações) e formas de uso. Métodos: Será feita uma revisão ampla da literatura em bases de dados, de informações médicas e de artigos científicos, conhecidas da comunidade científica, como o Uptodate ${ }^{\circledR}$, Dynamed $^{\circledR}$, Scielo ${ }^{\circledR}$, PubMed $^{\circledR}$, Google Scholar ${ }^{\circledR}$, entre outras, a fim de se compilar as

\footnotetext{
${ }^{1}$ Acadêmico de medicina / Faculdade de Medicina da Universidade Federal de Minas Gerais (UFMG). E-mail: thiagoamg5@gmail.com.

${ }^{2}$ Acadêmico de medicina / Faculdade de Medicina da Universidade Federal de Minas Gerais (UFMG)

3 Acadêmico de medicina / Faculdade de Medicina da Universidade Federal de Minas Gerais (UFMG)

${ }^{4}$ Faculdade Ciências Médicas de Minas Gerais (FCMMG)

5 Acadêmico de medicina / Faculdade de Medicina da Universidade Federal de Minas Gerais (UFMG)

${ }^{6}$ Acadêmico de medicina / Faculdade de Medicina da Universidade Federal de Minas Gerais (UFMG)

7 Acadêmico de medicina / Faculdade de Medicina da Universidade Federal de Minas Gerais (UFMG)

${ }^{8}$ Acadêmico de medicina / Faculdade de Medicina da Universidade Federal de Minas Gerais (UFMG)

9 Acadêmico de medicina / Faculdade de Medicina da Universidade Federal de Minas Gerais (UFMG)

ro Acadêmico de medicina / Faculdade de Medicina da Universidade Federal de Minas Gerais (UFMG)
} 
melhores evidências e indicações para cada tratamento farmacológico, os descritores utilizados nas pesquisas foram "fármaco em questão" AND "treatment of COVID-r9". Resultados: Dentre as atuais recomendações: demonstrou-se benefício que superam possíveis riscos para o uso de Dexametasona em pacientes que precisem de suporte de oxigênio; o toxilizumab tem sua recomendação de uso com benefícios em pacientes que evoluem para o estado grave ou crítico, ou para aqueles que persistem com marcadores inflamatórios altos apesar da terapia com dexametasona; o redemsivir também demostrou seu maior benefício clínico em pacientes hospitalizados, sob oxigenoterapia de baixo fluxo; a associação bamlavizumab/etesevisumab demostrou possível benefício para pacientes com a forma leve da doença que apresentem condições de risco para desfecho clínico desfavorável. Dentre os outros fármacos, não indicação específica para seu uso, ou carecem de mais ensaios clínicos de qualidade. Conclusões: Foram encontradas recomendações formais de uso de alguns fármacos para o tratamento da COVID-ı9, como a Dexametasona, o Tocilizumab, Redemsivir e bamlavizumab/etesevisumab, enquanto outros carecem de estudos mais robustos para uso fora do contexto de ensaios clínicos.

Palavras-chave: Tratamento farmacológico. COVID i9. Evidências.

ABSTRACT: Introduction: The COVID ig Pandemic brought, in a short period of time, a large number of infected patients with about $20 \%$ of them evolving to the severe or critical form of the disease, requiring hospitalization (WHO, 2021). The great search for health services and the high number of deaths brought haste to science for the discovery of possible medications and therapeutic actions that would mitigate the harmful consequences of the pandemic. Objectives: Thus, the present study lends itself to discussing, in the light of the best evidence and recommendations, the main drugs analyzed in the treatment of the disease (dexamethasone, tocilizumab, remdesivir, immunoglobulin, ivermectin and hydroxychloroquine, among others), their indications (or contraindications) ) and forms of use. Methods: A comprehensive review of the literature will be carried out on databases, medical information and scientific articles, known to the scientific community, such as Uptodate $^{\circledR}$, Dynamed ${ }^{\circledR}$, Scielo $^{\circledR}$, PubMed $^{\circledR}$, Google Scholar ${ }^{\circledR}$, among others, in order to After compiling the best evidence and indications for each pharmacological treatment, the descriptors used in the research were "drug in question" AND "treatment of COVID-I9". Results: Among the current recommendations: benefits have been demonstrated that outweigh possible risks for the use of Dexamethasone in patients who need oxygen support; toxilizumab is recommended for use with benefits in patients who progress to a severe or critical condition, or to those who persist with high inflammatory markers despite dexamethasone therapy; redemsivir also demonstrated its greatest clinical benefit in hospitalized patients, under low flow oxygen therapy; the bamlavizumab / etesevisumab association demonstrated a possible benefit for patients with the mild form of the disease who present risk conditions for an unfavorable clinical outcome. Among other drugs, there is no specific indication for their use, or they need more quality clinical trials. Conclusions: Formal recommendations were found for the use of some drugs for the treatment of COVID-19, such as Dexamethasone, Tocilizumab, Redemsivir and bamlavizumab / etesevisumab, while others lack more robust studies for use outside the context of clinical trials.

Keywords: Pharmacological treatment. COVID-ig. Evidence. 


\section{INTRODUÇÃO}

A Pandemia de COVID ig trouxe, em curto espaço de tempo, um grande número de pacientes infectados com cerca de $20 \%$ deles evoluindo para a forma severa ou crítica da doença, com necessidade de internação hospitalar (WHO, 202I). A grande busca por serviços de saúde e o elevado número de mortes trouxe pressa à ciência para o descobrimento de possíveis medicamentos e ações terapêuticas que mitigassem as consequências danosas da pandemia. Enquanto uma imunização em massa da população não se mostra amplamente disponível, vários fármacos foram (e vem sendo) estudados para serem empregados de maneira segura $\mathrm{e}$ possivelmente reduzir a morbimortalidade de pacientes, principalmente para aqueles que evoluem de maneira desfavorável.

\section{Objetivos}

Assim, o presente estudo se presta a discutir, à luz das melhores evidências e recomendações, os principais fármacos analisados no tratamento da doença (dexametasona, tocilizumabe, remdesivir, plasma convalescente, ivermectina e hidroxicloroquina, entre outros), suas indicações (ou contraindicações) e formas de uso. A intenção é trazer de forma clara e compilada, as principais revisões a cerca dos diferentes tratamentos assim como as recomendações das mais diversas e importantes sociedades científicas do mundo.

\section{Métodos}

Será feita uma revisão ampla da literatura em bases de dados, de informações médicas e de artigos científicos, conhecidas da comunidade científica, como o Uptodate ${ }^{\circledR}$, Dynamed ${ }^{\circledR}$, Scielo ${ }^{\circledR}$, PubMed $^{\circledR}$, entre outras, a fim de se compilar as melhores evidências e indicações para cada tratamento farmacológico. Serão descritas separadamente, por fármaco, as análises dos resultados encontrados em diversos ensaios clínicos e nas das discussões das principais bases de evidências médicas disponíveis.

\section{Resultados}

\section{Dexametasona/glicocorticoides}

Tais fármacos são ótimos agentes anti-inflamatórios, agem em diferentes nichos do sistema imune. Diminuem a expressão de diversos fatores inflamatórios, agindo tanto no sistema imune inato quanto ao adaptativo (RANG et al., 20I6). A fisiopatologia da síndrome do desconforto respiratório agudo (SDRA) envolve grande reação inflamatória pulmonar, evoluindo com alteração anatômica e progressiva piora do quadro. Assim, se justifica a busca pelo uso dos glicocorticoides, no entanto seu uso é controverso na literatura. A utilização é indicada para a SDRA inicialmente refratária e para indicações independentes da SDRA, usualmente não indicada para acometimentos virais (MATTHAY et al., 2019).

Os primeiros resultados sobre o efeito dos glicocorticoides foram conhecidos por dados preliminares do grupo de estudos inglês RECOVERY, o qual em junho de 2020 mostrou diminuição da mortalidade em pacientes com necessidade de suporte ventilatório. A partir do exposto diversos órgãos como: FDA (U.S. Food and Drugs Administration), EMA (European Medicines Agency) e no Brasil a ANVISA (Agência Nacional de Vigilância Sanitária) possibilitam seu uso em casos selecionados.

As meta-análises mais recentes confirmam o já exposto como resultado preliminar, ainda no primeiro semestre de 2020. Afirmando o benefício visto na mortalidade em pacientes 
com importante acometimento da doença. Estudos confirmaram, até então, a falta de benefício no uso preventivo, precoce ou em apresentações mais leves da doença.

Em meta-analise que contou com 7 estudos totalizando 1703 pacientes acometidos por quadro graves confirmados, o uso de glicocorticoides sistêmicos (dexametasona, hidrocortisona e metilpredinisolona) aumentou a sobrevida em média do grupo medicado em comparação ao grupo placebo em 28 dias (32\% versus 40\%, OR o,66, IC 95\% 0,53-0,82) (REACT, 2020).

Em outra meta-analise que computou 44 estudos, e mais de 20 mil pacientes, o uso dos glicocorticoides demonstrou-se significativo na diminuição da mortalidade (OR 0,72, IC 95\% o,57-0,87), assim como na diminuição de necessidade de ventilação mecânica (RR o,7I, IC 95\% 0,54-0,97) (VAN PAASSEN et al., 2020).

Parte importante destes dados vem do grupo de estudos britânico já citado: RECOVERY. Neste estudo, houve diminuição da mortalidade de grupos acometidos com manifestação severa da doença, os que necessitavam de suporte ventilatório. Em contrapartida, pacientes com acometimento leve da doença tiveram aumento da mortalidade sem validade estatística (17,8\% versus 14,0\%; RR I,I9; IC 95\%, o,92-1,55) (RECOVERY COLLABORATIVE GROUP, 2021b).

Em sinergia a contraindicação de uso de glicocorticoides utilizados em apresentações de doença que não necessitem de suplementação de oxigênio, já existem indicativos que não só a falta de benefício é encontrada como também o aumento de mortalidade como em meta-análise de Pasin et al. (2020), (17\% versus 13\%, RR I,23, IC 95\%, I,00-1,62).

Em suma, há evidências robustas do uso de glicocorticoides em pacientes que necessitem de suporte ventilatório, não sendo indicada e até restringida em pacientes sem necessidade de medidas ventilatórias ou seja, em provável acometimento leve/moderado da doença.

\section{Tocilizumabe}

Tocilizumabe é um fármaco que atua na inibição do receptor de interleucina 6 (IL-6), o qual é utilizado para tratamento de Artrite Reumatoide, Arterite de Células Gigantes e Síndrome de Liberação de Citocinas (SCOTT, 2017). No contexto da COVID-I9 foram identificadas correlações entre níveis aumentados de IL-6 associado a contextos clínicos de maior gravidade e mortalidade (HEROLD, 2020). Nesse sentido, diversos estudos objetivaram identificar se a utilização do Tocilizumabe no tratamento da COVID-I9 influencia em obtenção de melhores desfechos clínicos.

Um ensaio duplo-cego, randomizado, que envolveu 243 pacientes com COVID-I9 grave que não estavam em uso de suporte ventilatório, demonstrou não haver diferença na taxa de intubação ou morte a partir de utilização de dose única de Tocilizumabe em comparação com placebo (Io,6\% versos I2,5\%, HR o,83, IC 95\% 0,38-1,8I) (STONE et al., 2020).

Em outro estudo que incluiu 389 pacientes hospitalizados com COVID-rg que não estavam em suporte ventilatório, o tocilizumabe reduziu a progressão para ventilação mecânica ou morte em 28 dias (12\% contra 19\%; HR 0,556, IC 95\% 0,33-0,97), no entanto não reduziu a mortalidade geral em 28 dias (Io,4\% contra 8,6\%) (SALAMA, et al., 2020).

Um estudo randomizado aberto, realizado no Brasil que incluiu 129 pacientes, também não mostrou redução de risco de ventilação mecânica ou morte em 15 dias (28\% versus 20\%, OR I,54, 95\% 0,7-3,7), e houve uma tendência de maior mortalidade em 28 dias com tocilizumabe (2I versus 9 por cento, OR 2,7, IC 95\% 0,97-8,35) (VEIGA et al.,2021).

De acordo com os resultados preliminares não publicados de um estudo aberto realizado no Reino Unido, que incluiu 4II6 pacientes com quadro suspeito ou confirmado de COVID-I9, 
demonstrou que a utilização do Tocilizumabe com base no peso reduziu a taxa de mortalidade de 28 dias em comparação com o tratamento usual sozinho (29 versus $33 \%$, RR o,86, IC 95\% o,77-0,96) (PETER et al., 202I).

Os resultados preliminares de outro ensaio clínico randomizado internacional aberto que incluiu 803 pacientes com COVID-19 grave, que haviam sido internados em UTI e necessitaram de suporte respiratório ou cardiovascular, sugeriram um benefício na redução da mortalidade a partir da utilização dos inibidores da via da IL-6 Tocilizumabe e Salirumabe. Tocilizumabe $(n=353)$ e Sarilumabe $(n=48)$ reduziram a mortalidade intra-hospitalar em comparação com o atendimento padrão (28 e 22 versus $36 \%$; OR ajustada para sobrevivência hospitalar I,64, IC 95\% [CrI] I,I4-2,35 para Tocilizumabe e 2,0I, 95\% CrI 1,18-4,7I para Sarilumabe (GORDON et al., 2021).

Atualmente não há razões que justifiquem os diferentes desfechos encontrados. Os estudos que demonstram algum potencial benefício do uso do Tocilizumabe estão mais associados com uso concomitante de glicocorticoide, no entanto não foi bem estabelecido se esta associação resulta em alguma implicação. Apesar dos desfechos diversos os grupos de especialistas e as diretrizes governamentais como National Institutes of Health (NIH), Infectious Diseases Society of America (IDSA) e National Health Service (NHS) pouco diferem quando as recomendações sobre a utilização do Tocilizumabe e sugerem sua associação com dexametasona em pacientes em estado grave ou crítico e que possuem níveis séricos aumentados de marcadores inflamatórios (NIH, 202Ic; IDSA, 2021; NHS, 2021).

\section{Redemsivir}

O fármaco Remdesivir é um antiviral estudado inicialmente em meio aos surtos de Ebola na África Ocidental entre 2013 e 2016 sendo posteriormente verificada sua eficácia in-vitro tanto para os vírus Marbung, quanto as famílias de filovírus, como pneumovírus, paramixovírus e coronavírus (WARREN et al., 2016; LO et al., 2017). Como representa um análogo de adenosina parenteral, seu mecanismo de ação reside na inibição da RNA polimerase dependente de RNA viral, visando interromper a replicação do genoma viral (CARESTIATO et al., 2020).

No contexto do surgimento de uma nova doença relacionada ao coronavírus, o SarsCov-2 (COVID-19), em dezembro de 2019 na china até os dias atuais a comunidade científica se voltou para as diversas terapias que pudessem impactar no tratamento dos pacientes, visando reduzir mortalidade, morbidade, tempo de internação e de eliminação do vírus. O Remdesevir foi avaliado exclusivamente em pacientes hospitalizados, entre eles os pacientes com quadro grave e também com quadro moderado ou leve, sendo analisados considerando necessidade de suporte ventilatório, de alto ou baixo fluxo, ventilação invasiva e ECMO (Membrana de Oxigenação Extracorpórea).

Estudo denominado SOLIDARITY patrocinado pela OMS (Organização Mundial de Saúde), realizado com pacientes hospitalizados não encontrou diferença de mortalidade geral em 28 dias entre os 2750 pacientes alocados aleatoriamente em uso de terapia com Remdesivir e os 2708 pacientes com padrão de cuidados de suporte habitual (WHO, 202I). Outra referência que foi o estudo NIADI-ACTT-I, randomizado e controlado com 1062 pacientes, observou redução de tempo para alta e saída do suporte ventilatório de is para io dias, porém restrita para pacientes com suporte ventilatório de baixo fluxo ou não invasivo. Além disso, encontrou redução da mortalidade em 29 dias também restrita ao grupo com suporte de baixo fluxo e não invasivo, o que denota benefícios inexistentes ou pouco 
significativos em pacientes em ECMO e ventilação de alto fluxo ou invasiva (BEIGEL et al., 2020).

Em vista dos resultados desses dois estudos citados, a OMS acabou por recomendar contra o uso do Remdesivir, além do padrão habitual de cuidados, independente da gravidade da doença (WHO, 2020; LAMONTAGNE et al., 2020), o que diverge das conclusões e recomendações de outras sociedades e instituições, baseadas também em outros estudos. $O$ estudo SIMPLE-I, randomizado controlado que contou com 402 pacientes hospitalizados com mais de 12 anos e COVID-I9 confirmado, observou melhora clínica com uso de Remdesivir por 5 ou Io dias e redução de eventos adversos graves no grupo que utilizou o fármaco. Além dele o SIMPLE-2 randomizado e controlado, analisou 596 adultos e crianças também acima dos I2 anos e hospitalizados com COVID moderada, e concluiu que o tratamento de cinco dias com Remdesivir pode aumentar a probabilidade clínica de melhora, sendo assim ambos os estudos apoiaram a recomendação da FDA (U.S. Food And Drug admnistration) do uso do medicamento em pacientes com mais de 12 anos e com $40 \mathrm{~kg}$ ou mais que requerem hospitalização.

Nessa mesma linha, o NIH (National Institute of Health) dos E.U.A, recomendou o uso do Remdesivir em pacientes hospitalizados e em uso de oxigênio suplementar, a associação do Remdesivir com Dexametasona em pacientes com oxigênio suplementar de alto fluxo ou ventilação invasiva e duração do tratamento de 5 dias ou até a alta hospitalar, podendo se estender por io dias(NIH, 202Ia). Devemos citar também a IDSA (Infectious Diseases Society of America) que recomenda o Remdesivir em pacientes hospitalizados com doença grave, onde o curso do tratamento é de cinco dias em pacientes com oxigênio de baixo fluxo e Io dias nos pacientes em ventilação invasiva e ECMO, e ainda indica contra o uso rotineiro do fármaco em pacientes hospitalizados sem necessidade de oxigênio ou saturando mais que $94 \%$ em ar ambiente.

No Brasil as perspectivas sobre o tratamento da COVID-ıg tiveram uma mudança com a aprovação do Remdesivir no dia 12 de março de 202I para uso em pacientes hospitalizados pela ANVISA (Agência Nacional de Vigilância Sanitária). Em meio ao cenário de imunização de pequena porcentagem da população, do surgimento de novas variantes e de grande aumento de casos e mortes, a inserção de novas terapias ganha protagonismo, mesmo em situações que ainda necessite de mais evidências.

Concluindo podemos ver que a principal indicação do Remdesivir será em pacientes hospitalizados com suporte de oxigênio de baixo fluxo, e que seu uso em pacientes hospitalizados graves de rotina ou que foram intubados há pouco tempo ( 24 à $48 \mathrm{~h}$ ), tem benefício clínico incerto. Devemos citar que o uso de Remdesivir com Hidroxicloroquina e cloroquina é contraindicado pela interação medicamentosa existente e que o Baricitinibe (Inibidor da Janus Quinase) é uma opção de uso em associação com o Remdesivir em pacientes alérgicos à Dexametasona ou em ausência da mesma, isso em quadros graves, com necessidade de suporte ventilatório de baixo fluxo, ventilação invasiva ou ECMO (KALIL, 2020).

\section{Plasma convalescente}

Plasma convalescente é uma estratégia de imunização passiva que consiste na utilização de anticorpos neutralizantes obtidos de indivíduos que já foram infectados e se recuperaram. Essa terapêutica, ao reduzir a entrada do vírus nas células e aumentar o clearance viral tem o potencial teórico de reduzir o risco de infecção e a gravidade das manifestações. (NGUYEN et al., 2020). 
Antes da COVID-ig, o uso do plasma convalescente no tratamento de síndrome respiratória aguda grave de etiologia viral já havia sido analisado em uma meta-análise britânica com 32 estudos observacionais, na qual evidenciou-se redução de mortalidade (OR o,25; IC 95\% 0,14-0,45; $\mathrm{I}^{2}=0 \%$ ), especialmente quando a terapia foi administrada precocemente após o início dos sintomas. (MAIR-JENKINS et al., 2015)

Essa terapia aplicada ao tratamento do Novo Coronavírus ganhou extrema notoriedade após autorização de uso emergencial pela agência regulatória americana em agosto de 2020 (U.S. FOOD AND DRUG ADMINISTRATION, 2020). Vale ressaltar que foi autorizado o uso em condições específicas: plasma convalescente com altos títulos de anticorpos neutralizantes, em pacientes hospitalizados por COVID-I9, no início do curso da doença ou que tenham imunidade humoral deficiente. Os eventos adversos graves associados ao tratamento são raros e consistem principalmente de reações transfusionais, complicações tromboembólicas e eventos cardíacos. Sendo a maioria das complicações cardíacas e tromboembólicas não relacionadas ao tratamento com plasma (MAIR-JENKINS et al., 2020).

Apesar do grande potencial teórico e dos resultados promissores em estudos observacionais, os ensaios clínicos randomizados iniciais não demonstraram benefício clínico claro do uso da terapia em pacientes hospitalizados. (CHAI et al., 2020).

\section{Azitromicina}

Azitromicina é uma droga da classe dos macrolídeos, com conhecida ação antibiótica e imunomoduladora, além de possuir um perfil de segurança muito bem conhecido. Tendo em vista essas propriedades, é uma boa escolha para o tratamento de pneumonias bacterianas e seu uso no tratamento da COVID-ıg foi questionado e estudado por diversos grupos. Do ponto de vista teórico, a redução da produção de citocinas inflamatórias e inibição da ativação neutrofílica agiriam no componente inflamatório da doença, principal mecanismo por detrás do agravamento da infecção (RECOVERY COLLABORATIVE GROUP, 202ıa).

Pesquisas iniciais, in vitro, comprovaram a capacidade da azitromicina em inibir a replicação do SARS-COV2. A concentração do fármaco nesse estudo é passível de ser alcançada nos pulmões com doses de $500 \mathrm{mg}$ diárias da medicação, o que gerou entusiasmo na comunidade científica e embasou a realização de uma série de estudos clínicos muito robustos, tendo em vista o refinamento metodológico e amostral (ECHEVERRÍA-ESNAL, 2020).

A iniciativa RECOVERY, conduzida pelo NIH inglês em conjunto com a universidade de Oxford, buscou avaliar uma série de tratamentos no contexto da COVID-ig. Nesse sentido, a azitromicina foi estudada através de um estudo randomizado, controlado e aberto em que 7763 doentes hospitalizados por suspeita de COVID-I9 ou doença confirmada laboratorialmente foram divididos entre um grupo controle (em que o tratamento padrão era sintomático e suportivo) e um grupo em que a intervenção era o tratamento padrão associado a administração por ıo dias de $500 \mathrm{mg}$ diárias do fármaco. O desfecho primário analisado foi mortalidade global em 28 dias, em que não houve diferenças entre o grupo placebo e controle. Os desfechos secundários (tempo de permanência hospitalar, necessidade de ventilação mecânica e morte por COVID-ı) também não demonstraram que o macrolídeo em questão possui benefícios em relação ao tratamento padrão (RECOVERY COLLABORATIVE GROUP, 2021).

Outros estudos, com menor poder estatístico, também buscaram avaliar a eficácia da terapia com azitromicina na COVID-I9. O estudo multicêntrico PRINCIPLE distribuiu de forma aleatória 1362 pessoas com suspeita ou confirmação de COVID-I9, e, como no estudo 
anterior, tinha como proposta uma dosagem diária de $500 \mathrm{mg}$ de azitromicina, porém por 3 dias. O grande diferencial desse trabalho foi selecionar uma amostra proveniente da comunidade e com risco aumentado para desfechos desfavoráveis secundários à COVID-I9, tais como idosos com mais de 65 anos ou aqueles maiores de 50 anos e com comorbidades associadas. Os desfechos primários foram a mortalidade, internações hospitalares e relato subjetivo de melhora dos sintomas. Nenhum desses parâmetros foi afetado de forma substancial pela intervenção farmacológica, de modo com que os autores da pesquisa também corroboram com a não utilização dessa medicação como uma rotina nos casos de COVID-I9 (PRINCIPLE TRIAL COLLABORATIVE GROUP, 202I).

Um estudo brasileiro denominado COALITION II buscou estudar o efeito da terapia com azitromicina nos pacientes com manifestações respiratórias graves da COVID-i9. Dessa forma, com uma amostra de 397 pacientes, comparou-se o tratamento padrão (diferentemente dos demais estudos, o tratamento padrão na ocasião previa uso de $400 \mathrm{mg}$ duas vezes ao dia de hidroxicloroquina por um período de io dias) com o padrão associado ao macrolídeo. $O$ desfecho primário foi dado pela avaliação de uma escala objetiva de status clínico após is dias do início da terapia, já o desfecho secundário mais importante no estudo foi a mortalidade após 29 dias de intervenção. Quando comparado ao tratamento padrão, o uso da azitromicina não se associou a nenhuma diferença nos desfechos estudados (FURTADO, 2020).

O uso de azitromicina como terapia padrão para casos de COVID-I9, seja a doença leve ou grave, não se mostrou benéfica em termos de desfechos clínicos. Tendo em vista seus potenciais malefícios, principalmente relacionados à aquisição de resistência bacteriana, a terapia com o macrolídeo em questão deve ser restrita apenas para aqueles doentes com suspeita de infecção bacteriana associada.

\section{Hidroxicloroquina/cloroquina}

A Cloroquina é uma droga antimalárica, da classe das 4-aminoquinolinas, desenvolvida em 1934. A Hidroxicloroquina, sua análoga, foi desenvolvida em ra46 e é utilizada no tratamento de doenças autoimunes como o Lúpus Eritematoso Sistêmico (LES) e a Artrite Reumatóide, assim como no tratamento da malária.

Sabe-se que ambas as drogas possuem a capacidade de elevar o $\mathrm{pH}$ endossomal e, consequentemente, inibir a fusão do SARS-CoV-2 com a membrana celular do hospedeiro, além de interferir na ligação do vírus com o receptor celular pela inibição da glicosilação da enzima conversora de angiotensina-2 (ECA-2), a qual é fundamental neste processo químico (RECOVERY COLLABORATIVE GROUP, 2020). Estudos in vitro demonstraram que tanto a Cloroquina quanto a Hidroxicloroquina bloqueiam a atividade do SARS-CoV-2 prevenindo a liberação do genoma viral (LIU et al., 2020). Além de tais mecanismos, uma consideração gerada pela comunidade científica é a de que, por ambas as drogas possuírem caráter imunomodulador, elas poderiam ser capazes de auxiliar nos mecanismos inflamatórios da doença e corresponder em seu tratamento. Apesar de tantas teorias e hipóteses acerca da eficácia desses medicamentos no tratamento da Covid-I9, os mais recentes estudos e ensaios clínicos comprovam, por hora, a falta de evidência de que Cloroquina e Hidroxicloroquina possam vir a tratar a doença.

Em um estudo randomizado, controlado e aberto realizado pela iniciativa RECOVERY, no Reino Unido, a Hidroxicloroquina não reduziu a mortalidade em 28 dias quando comparada ao tratamento usual do grupo controle (baseado no manejo de sintomas e suportivo) em pacientes hospitalizados. Curiosamente, os pacientes tratados com a Hidroxicloroquina apresentaram maior tempo de internação e, dentro daqueles pacientes que não foram 
submetidos à ventilação, apresentaram maior risco de ventilação mecânica e óbito, comparados àqueles submetidos ao tratamento padrão (RECOVERY COLLABORATIVE GROUP, 2020).

No Brasil, Cavalcanti et al. conduziram um ensaio controlado, randomizado, multicêntrico e aberto em 55 hospitais envolvendo pacientes hospitalizados nos quais a Hidroxicloroquina isolada ou em combinação com Azitromicina não resultou em melhores desfechos clínicos para os pacientes. Ademais, percebeu-se que eventos adversos como elevações de enzimas hepáticas e prolongação do intervalo-QT foram mais comuns em pacientes submetidos ao tratamento alternativo quando comparado ao padrão (CAVALCANTI et al., 2020)

Estudou-se também a possibilidade de prevenção da Covid-ı́ a partir do uso de Hidroxicloroquina em pacientes pós-exposição ao SARS-CoV-2. Neste contexto, a droga funcionaria como um método profilático para que a doença não evoluísse após a contaminação. Um ensaio randomizado e aberto foi realizado na Espanha envolvendo indivíduos assintomáticos que tiveram contato com pacientes que apresentaram RT-PCR (Reverse Transcription-Polymerase Chain Reaction) positivo. A análise incluiu 2314 indivíduos, divididos em 2 grupos, em que um grupo receberia a droga e o outro receberia o cuidado padrão (sem terapia específica). O desfecho primário foi a confirmação do PCR e a Covid-ı9 sintomática em 14 dias. Os resultados se mostraram similares quanto à incidência da doença $e$ confirmação do RT-PCR (5,7\% e 6,2\%; razão de risco o,86; intervalo de confiança de 95\%, o,52 a $\mathrm{I}, 42$ ). Além disso, a Hidroxicloroquina apresentou maior incidência de efeitos adversos ( $56,1 \%$ x 5,9\%) e não esteve associada à menor incidência de transmissão do SARS-CoV-2 (MITJÁ et al., 2020).

Estudo semelhante foi realizado nos Estados Unidos e Canadá, através de um ensaio randomizado, duplo-cego e controlado testando a Hidroxicloroquina como um profilático pósexposição. Um grupo de pacientes recebia placebo, enquanto o outro era submetido à droga. $\mathrm{O}$ desfecho primário era a incidência de, ou a confirmação laboratorial de Covid-ı, ou sintomas compatíveis com a doença em I4 dias. Dos 821 pacientes participantes, 87.6\% reportaram a exposição de alto risco pelo contato com indivíduo confirmadamente diagnosticado com a doença. A incidência do aparecimento da doença nestes pacientes não foi significamente diferente entre os dois grupos $(2,4 \%$ de diferença, IC $95 \%, P=0,35)$. Novamente os efeitos colaterais foram maiores no grupo em que foi administrada a Hidroxicloroquina (40,1\% $\mathrm{x}$ $\mathrm{I} 6,8 \%)$. O estudo mostrou, mais uma vez, que o medicamento não previne a doença (BOULWARE et al., 2020).

Conclui-se, a partir de todos os estudos supracitados, que não foram encontradas evidências suficientes que demonstrassem a eficácia da Hidroxicloroquina, tanto na prevenção quanto no tratamento da Covid-ı. Reiteramos que há espaço para que novos estudos sejam realizados para a possível aplicação deste medicamento nos pacientes da Covid-I9, mas que, por hora, não há recomendações científicas para tal. Partindo disso, o renomado Painel de Diretrizes de Tratamento do Covid-ı9, realizado pela NIH (National Institutes of Health), dos Estados Unidos, orienta para a não utilização do medicamento (NIH, 2021).

\section{Ivermectina}

A ivermectina é um fármaco anti-helmíntico que foi introduzido no mercado em 198I, primeiramente para uso veterinário. Trata-se de uma droga muito segura, usada como tratamento de primeira linha em muitas infecções filariais. Em termos bioquímicos, é um agente semissintético derivado de substâncias naturais, as avermectinas, obtidas a partir de um 
microorganismo actinomicético. A forma de administração é oral e o medicamento possui meia vida de Ir horas. (RANG et al., 2016)

Alguns resultados preliminares com essa droga, principalmente em estudos em vitro, mostraram resultados que apesar de pouco conclusivos, chamaram bastante atenção em meio ao contexto de corrida global por um medicamento eficaz contra a infecção pelo SARS-CoV-2. Em estudos recentes, a ivermectina demonstrou a capacidade in-vitro de inibir a replicação de alguns virus de RNA de fita simples, como virus da Dengue, zika virus, virus da febre amarela e outros. (CHACCOUR et al. 2020)

Recentemente Caly et al. reportou um potencial benefício da ivermectina no tratamento da COVIDig, o que gerou repercussão imediata da comunidade científica. O estudo foi realizado in vitro, onde a droga demonstrou ser um potente inibidor da replicação viral do SARS-CoV-2. (CALY et al., 2020) No entanto, nesse experimento a dosagem utilizada foi o equivalente a 50 a roo vezes a concentração máxima obtida no plasma de um ser humano após a administração de uma dose única de $200 \mu \mathrm{M} / \mathrm{kg}$ da ivermectina (dose normalmente usada para o controle da Oncocercose). (CHACCOUR et al., 2017)

Além dos resultados ainda inconclusivos a respeito da real eficácia da ivermectina, já existem outros estudos que advogam contra o seu uso de maneira "off-label" para o tratamento da COVIDig. Estudos em modelos animais demonstraram potencial risco de neurotoxicidade. (MÉNEZ, 2012) Tal efeito adverso é facilmente prevenido pela presença de barreira hematoencefálica (BHE) intacta e funcionante, no entanto pacientes em estado hiperinflamatório podem apresentar aumento da permeabilidade endotelial na BHE, levando a uma possível predisposição a eventos neurotóxicos (VARATHARAJ, 2017; POYIADJI, 2020) Além disso, alguns antirretrovirais que veem sendo utilizados no tratamento da COVID-I9 são potentes inibidores do Citocromo $\mathrm{P}_{45}{ }_{3} \mathrm{~A}_{4}\left(\mathrm{CYP}_{3} \mathrm{~A}_{4}\right)$, a principal via de metabolização da ivermectina, o que aumentaria a biodisponibilidade desse fármaco de maneira indesejada. $\mathrm{E}$, por fim, de acordo com as evidências que existem até o momento os níveis de ivermectina com atividade efetiva contra o SARS-CoV-2 não seriam alcançadas sem doses extremamente altas com potencial aumento da toxicidade. (CHACCOUR, et al., 2020)

Em contrapartida, evidências de estudos recentes em modelos animais demonstraram que uma semana após a administração do medicamento por via oral, foram encontrados níveis de ivermectina três vezes maiores no tecido pulmonar do que no plasma. Resultados como esse deixam a porta aberta para pesquisas futuras, em particular para o uso da ivermectina no tratamento das infecções virais das vias aéreas. (LESPINE et al., 2005; CHIU et at., 1989)

Até o momento, a grande maioria das diretrizes contra a COVIDig não recomenda o uso da ivermectina no tratamento da COVID-ı. A Infectious Diseases Society of America não recomenda o uso desse fármaco fora do contexto de ensaios clínicos para o tratamento da COVID-ı9 (IDSA, 2021). Já o National Institutes of Health declara que à luz das evidências atuais não há possibilidade de se advogar a favor ou contra o uso desse medicamento e que mais estudos são necessários para o esclarecimento. (NIH, 2021). As diretrizes para tratamento e diagnóstico da COVIDı́ do Ministério da Saúde publicadas no dia o7 de maior de 2020 declara que ainda não existem evidência clínicas suficientes para fazer qualquer recomendação a respeito do uso da ivermectina para o tratamento da COVID-19. (Diretrizes para diagnóstico e tratamento da COVID-19 versão 04, 2020)

\section{Heparina/outros anticoagulantes}

Em um estudo coorte realizado em abril/2020 com 184 pacientes, demonstraram a prevalência de eventos trombóticos em 31\% dos pacientes com Covid-19 admitidos na unidade 
de tratamento intensivo (UTI), incluindo trombose venosa profunda (TVP) e trombose arterial, na ordem de $27 \%$ e $3,7 \%$, respectivamente, tendo a TEP como complicação mais frequente a despeito da tromboprofilaxia padronizada adequada em todos os pacientes (KLOK, 2020). Em outro estudo post-mortem, as necropsias de pacientes que morreram com Covid-19 demonstraram presença de trombos na vasculatura pulmonar com frequência nove vezes maior do que a de pacientes com síndrome respiratória aguda grave (SARS) por influenza. (ACKERMANN, et al., 2020) Embora ainda não existam evidências concretas acerca da incidência dos eventos trombóticos em pacientes com Covid-I9, eventos dessa natureza são marca registrada da infecção (OXLEY, et al., 2020) e estratégias terapêuticas adequadas continuam em atualização.(CHEST, 2020)

A fisiopatologia sobre como a doença promove os eventos trombóticos não é bem elucidada mas os pulmões dos pacientes com Covid-ı demonstram todos os pilares da tríade de Virchow: hipercoagulabilidade, lesão endotelial e estase sanguínea. São observados nesses pacientes em estágios iniciais da coagulopatia níveis elevados de fibrinogênio, dímero-d, angiopoietina II, fator VIII, fator de Von Willebrand além de existirem evidências de lesão endotelial direta do vírus ao infectar as células. (ACKERMANN et al., 2020; CONNORS, et al. 2020) Nesse contexto, foi observado que valores elevados de dímero-d se relacionam com prognósticos piores. (TANG et al., 2020)

A apresentação clínica inicial difere da coagulação intravascular disseminada (CIVD) por não apresentar sangramentos. Além disso, o tempo de tromboplastina parcial ativada, o tempo de protrombina e a contagem de plaquetas não se apresentam tão alterados e não são marcadores fidedignos de gravidade como na coagulopatia induzida por sepse. (TANG et al., 2020) Contudo, foi visto que existe evolução como em um espectro para a CIVD. Nesse contexto, a Sociedade Internacional de Trombose e Hemostasia (ISTH) recomenda a monitorização do tempo de tromboplastina parcial ativada, tempo de protrombina, contagem de plaquetas e de fibrinogênio, tal qual é recomendado para CIVD.

A Sociedade Americana de Hematologia (ASH), o Colégio Americano de Médicos do tórax (CHEST) e a ISTH recomendam o tratamento com anticoagulantes em doses profiláticas para todos os pacientes admitidos no hospital, incluindo UTI, e que não possuam contraindicações - sangramento ativo, plaquetas abaixo de 2500o. Não existem evidências de boa qualidade que sustentem ou contra indiquem o uso de dose terapêutica nos pacientes sem TEP ou TVP, sendo, portanto, recomendado a individualização de cada caso, avaliando o risco de trombose em contraponto ao risco de sangramento. A respeito da escolha do fármaco, apenas a CHEST recomenda o uso específico de heparina de baixo peso molecular (HBPM) ou fondaparinux em detrimento da heparina não fracionada (HNF), para pacientes internados na enfermaria; pacientes na UTI, orientam para HBPM e HNF em detrimento da fondaparinux. Justificam que, na ausência de evidências, o uso de HBPM traz menos exposição à equipe. Também advoga contra o uso dos novos anticoagulantes orais (NOACs) em qualquer nível de cuidado hospitalar por considerar alto o risco de rápido declínio clínico e as possíveis interações medicamentosas com demais medicações que o paciente possa estar em uso. A tromboprofilaxia mecânica deve ser usada apenas se houver contraindicação à terapia farmacológica. É importante notar que a lacuna de conhecimento sobre os mecanismos fisiopatológicos envolvidos na gênese trombótica e as poucas evidências de qualidade disponíveis dificultam a adoção de estratégias de cuidado bem estabelecidas e fundamentadas.

Para os pacientes com TVP ou TEP manifesta, a CHEST recomenda contra o uso generalizado de terapia trombolítica sistêmica, sendo esta resguardada aos pacientes com instabilidade hemodinâmica ou de disfunção cardíaca direita. Advoga contra, ainda, o uso de 
trombólise guiada por cateter em qualquer situação. $O$ tratamento anticoagulante desses pacientes deve dar preferência ao uso de HBPM ou fondaparinux, sendo o uso de HNF preconizado para pacientes com alto risco de sangramento, com descompensação hemodinâmica franca ou com probabilidade de descompensação por TEP e que a terapia de revascularização possa ser necessária. $O$ evento deve ser considerado como "TEP provocada" e a anticoagulação deve ser mantida por três meses com antagonistas de vitamina $\mathrm{K}$, NOACs ou HBPM. Para pacientes com TVP ou TEP tratados em regime ambulatorial, a terapia com NOACs pode ser utilizada na ausência de interações medicamentosas. Cabe ressaltar que não existe nenhum escore clínico para risco de sangramento validado para a Covid-I9 sendo, portanto, recomendado que seja utilizado o protocolo da instituição ou outros escores validados para coagulopatias.(CHEST, 2020)

Ao momento da confecção deste capítulo, vários ensaios clínicos estão em andamento para avaliar as principais lacunas de conhecimento, tais quais: a eficácia do uso de anticoagulação plena em detrimento da profilática; validação de escores de riscos para trombose e sangramento em pacientes com Covid-ı;; e, elucidação de biomarcadores fidedignos para maus desfechos. Destacamos os ensaios REMAP-CAP, ACTIV-4, and ATTACC (NCT 02735707,04505774 e 04372589, respectivamente). A atual conjuntura de esforços desmedidos para compreender e elaborar estratégias de cuidados ante o Sars-Cov-2 exige atualização contínua do conhecimento e é esperado que ocorram modificações nessas recomendações com o surgimento de melhores evidências. (CHEST, 2020)

\section{Outros}

Existem vários estudos em andamento sobre diversos outros medicamentos para possivel manejo da COVID-rg.

O fapiravir, inibidor da enzima RNA polimerase, usado em tratamento da influenza em alguns paises asiaticos demosntrou benefício em alguns ensaios (IVASHCHENKO et al., 2020), porem os fatores de confusão envolvidos, como a administração de outros agentes imunomoduladores faz com que esse resultado seja interpretado cautelosamente, não havendo indicação atual de uso terapêutico ou profilático (KIM, 202I).

Os interferons também entraram na lista de fármacos com possíveis benefícios, após ser verificado atividade em vitro contra o Sars-Cov-2 (CLEMENTI et al., 2020), todavia, até aqui, o maior estudo multinacional sobre o tema não evidenciou benefício (KIM, 202I).

Inibidores da interleucina I também estão sendo avaliados, como o anakira, que apresentou redução da taxa de mortalidade em pacientes internados inicialmente com ventilação não invasiva (CAVALLI et al., 2020). Todavia, estudos maiores e ramdomizados em pacientes leves e moderados não apresentou benefício (CORIMUNO-19, 202I).

O Lopinavir-ritonavir, apesar de apresentar atividade in vitro contra a COVID-I9 (GRONEBERG, 2005), também não demonstrou benefícios em pacientes hospitalizados, o uso do fármaco em casos leves e ambulatoriais não foi avaliado.

$\mathrm{O}$ uso dos anticorpos monoclonais bamlanivizimab mais etesevimabe é recomendado por algumas entidades, em caráter emergencial, para paciente leve/moderado, com risco aumentado para piora clínica, que não esteja em uso de oxigenio suplementar, em regime ambulatorial ( $\mathrm{NIH}, 202 \mathrm{Ib}$ ), na dose de 7oomg e r.40omg, respectivamente. Todavia, as 
recomendacoes apresentam ressalvas importantes, como dados incompletos sobre preditores de resposta clínica, ausência de dados sobre a eficácia da dose aprovada (que é diferente da dose utilizada no estudo original) pelo FDA (U.S Food and Drugs Administration).

A Suplementação com Vitanina $\mathrm{D}$ ganhou importância por sua possível participação no processo de imunidade contra o COVID-19. Alguns estudos interacionais associaram níveis séricos mais baixos de vitamina $\mathrm{D}$ aos pacientes internados em UTI, quando comparados aos níveis séricos de pacientes em enfermaria. Todavia, os fatores de risco identificados para carência da vitamina são, em grande parte, semelhantes a fatores de risco da COVID-I9, como diabetes e obesidade, tornando-os confundidores importantes nos estudos. Assim, com base nas evidencias disponíveis, a suplementação da vitamina continua apenas para indicaççoes formais, nos casos de carência identificada, e não para tratamento específico da COVID-I9 (KIM, 2021).

Estudos recentes também sugerem a suplementação de Zinco como alternativa terapêutica devido às propriedades imunomoduladoras e antivirais do mineral, ele tem o potencial de ser um tratamento de suporte em pacientes com COVID-ig. Foi sugerido que a suplementação de zinco pode aumentar a eficácia de outros tratamentos atualmente também sob análise, como a hidroxicloroquina. Foi demonstrado que a suplementação de zinco é capaz de diminuir os sintomas relacionados a COVID-19, como infecção do trato respiratório inferior. Sugere-se que esses efeitos são devidos à inibição da ligação e da replicação viral, e podem ser relevantes para COVID-19. Um ensaio clínico registrado na Austrália tem investigado o uso da administração intravenosa de zinco em indivíduos com COVID-I9 (SHAKOOR et al., 202I). A suplementação de zinco pode ter um efeito positivo durante a infecção por COVID-ı. No entanto, faltam ensaios clínicos acerca da terapêutica contra COVID-I9, assim o mineral não é indicado fora de ensaios clínicos.

A colchicina também foi colocada como um dos medicamentos com possível efeito terapêutico para a doença. Resultados de um estudo grego sugerem um aumento da sobrevida livre de doenças, no entanto, sem alterar mortalidade global. Ainda associou mais efeitos adversos, como a diarreia, ao uso do fármaco (DEFTEREOS et al., 2020). Assim, não há recomendação padrão para o uso da colchicina entre as diversas bases de evidencias médicas.

\section{CONCLUSÃO}

A pandemia de COVID-r9 está sendo, sem dúvida, um dos maiores desafios que humanidade e a ciência já enfrentaram no decorrer da história. Além do próprio vírus, diversas tensões em todos os âmbitos sociais e políticos eclodiram, levando ao questionamento do próprio conhecimento científico e intensificando a busca por soluções rápidas para o controle da doença. Nesse contexto, diversas pesquisas foram e estão sendo feitas para a busca de fármacos e de vacinas eficientes. Foram encontradas recomendações formais de uso de alguns fármacos para o tratamento da COVID-I9, como a Dexametasona, o Tocilizumab, Redemsivir e bamlavizumab/etesevisumab, enquanto outros carecem de estudos mais robustos para uso fora do contexto de ensaios clínicos. Assim, como em todos os tratamentos, é necessário seguir as evidências e o método científico, a fim de se estabelecer protocolos eficazes e seguros para todos os pacientes. 


\section{REFERÊNCIAS BIBLIOGRÁFICAS}

BOULWARE, D.R. et al. A Randomized trial of hydroxychloroquine as postexposure prophylaxis for covid-19. New England Journal of Medicine, v. 383, n. 6, p. 517-525, 2020.

CALY, L. et al. The fda-approved drug ivermectin inhibits the replication of sars-cov-2 in vitro. Antiviral Research, [S.L.], v. 178, p. 104787, 2020.

CAVALCANTI, A.B. et al. Hydroxychloroquine with or without azithromycin in mild-tomoderate covid-19. The New England Journal of Medicine, v. 383, n. 21, novembro de 2020, p. 204I-52. PubMed, doi:Io.1056/NEJMoazor9or4.

CAVALLI G, et al. Interleukin-I blockade with high-dose anakinra in patients with COVID19, acute respiratory distress syndrome, and hyperinflammation: a retrospective cohort study. Lancet Rheumatol 2020; 2:e325.

CHACCOUR, C. et al. Ivermectin and covid-I9: keeping rigor in times of urgency. The American Journal of Tropical Medicine and Hygiene, [S.L.], v. IO2, n. 6, p. II56-II57, 2020.

CHACCOUR, C. et al. Ivermectin to reduce malaria transmission I. Pharmacokinetic and pharmacodynamic considerations regarding efficacy and safety. Malaria Journal, [S.L.], v. I6, n. I, p. I6I, 2017.

CHAI, K.L. et al. Convalescent plasma or hyperimmune immunoglobulin for people with COVID-19: a living systematic review. The Cochrane database of systematic reviews v. Io, CDor36oo, 2020.

CHIU SH, L.A.Y. Metabolism and tissue residues. Ivermectin and Abamectin. SpringerVerlag, p.I3I-I43, I989.

CLEMENTI, $N$, et al. Interferon- $\beta$-ra inhibition of severe acute respiratory syndromecoronavirus 2 in vitro when administered after virus infection. Journal Infection Diseases, p.222:722, 2020.

CORIMUNO-19. Collaborative group. Effect of anakinra versus usual care in adults in hospital with covid-I9 and mild-to-moderate pneumonia (CORIMUNO-ANA-I): a randomised controlled trial. Lancet, p.9:295, 2021.

DEFTEREOS, S.G. et al. Effect of colchicine vs standard care on cardiac and inflammatory biomarkers and clinical outcomes in patients hospitalized with coronavirus disease 2019: the grecco-ı9 randomized clinical trial. JAMA network open, v. 3, n.6, p.ezor3136, 2020.

DYNAMED. Management of COVID-19. EBSCO Information Services, 2020. Disponível em: https://www.dynamed.com/management/management-of-covid-r9. Acesso em: 2 abr. 2021. 
ECHEVERRÍA-ESNAL, D. et. al Azithromycin in the treatment of covid-rg: a review. Expert Review of Anti-infective Therapy, v.19, p.147 - 163, 2021.

FURTADO, R.H.M. Azithromycin in addition to standard of care versus standard of care alone in the treatment of patients admitted to the hospital with severe COVID-I9 in Brazil (COALITION II): a randomised clinical trial. The Lancet, v. 396, p. 959 - 967, 2020.

GANDHI, R.T. The multidimensional challenge of treating coronavirus disease 2019 (covid19): remdesivir is a foot in the door. Clinical Infectious Diseases, 2020. Disponível em: https://academic.oup.com/cid/advance-article/doi/ı.1093/cid/ciaali32/5879440. Acesso em: 30 mar. 2021.

GORDON, A.C. et al. Interleukin-6 Receptor Antagonists in Critically Ill Patients with Covid-19. MedRxiv, p. 2021.01. 07.21249390, 2021.

GRIFFITH, B. Covid-I9-associated Acute Hemorrhagic Necrotizing Encephalopathy: imaging features. Radiology, [S.L.], v. 296, n. 2, p. II9-I20, 2020.

GRONEBERG, D.A. et al. Treatment and vaccines for severe acute respiratory syndrome. Lancet Infection Disease, v. 5, p.147, 2005.

HEROLD T. et al. Elevated levels of IL-6 and CRP predict the need for mechanical ventilation in COVID-19. Journal of Allergy and Clinical Immunology, v. I46, n. I, p. I28-136. e4, 2020.

IDSA. Infectious Diseases Society of America. Guidelines on the treatment and management of patients with covid-I9, 2021. Disponível em: https://www.idsociety.org/practiceguideline/covid-I9-guideline-treatment-and-management/. Acesso em: 30 mar. 202I.

IVASHCHENKO, A.A. et al. AVIFAVIR for treatment of patients with moderate covid-I9: interim results of a phase ii/iii multicenter randomized clinical trial. Clinical Infection Diseases, 2020.

KIM, A.Y. \& GANDHI, R.T. COVID-I9: Management in hospitalized adults. Em: Hirsch, MS, editor. UpToDate in Waltham, MA, 2021. Disponível em: https://www.uptodate.com/contents/covid-I9-management-in-hospitalized-adults. Acesso em: 30 mar. 2021.

LESPINE, A. et al. Influence of route of administration on efficacy and tissue distribution of ivermecvtin in goat. Vet Parasitology, v.128, p. 251-260, 2005.

LIU, J. et al. Hydroxychloroquine, a less toxic derivative of chloroquine, is effective in inhibiting sars-cov-2 infection in vitro. Cell Discovery, v. 6, p. 16, 2020.

MAIR-JENKINS J. et. al. Safety update: covid-19 convalescent plasma in 20,00o hospitalized patients. Mayo Clinic proceedings, v. 95, n.9, p.1888-1897, 2020.

MAIR-JENKINS, J. et al. Convalescent plasma study group. the effectiveness of convalescent plasma and hyperimmune immunoglobulin for the treatment of severe acute respiratory 
infections of viral etiology: a systematic review and exploratory meta-analysis. The Journal of infectious diseases, v.211, n.I, p. 80-90, 2015 .

MATTHAY, M.A. et al. Acute respiratory distress syndrome. Nature Reviews Disease Primers, v. 5, n. I, p. 18, 2019.

MÉNEZ, C. et al. Relative neurotoxicity of ivermectin and moxidectin in mdrab (-/-) mice and effects on mammalian gaba(a) channel activity. Plos Neglected Tropical Diseases, [S.L.], v. 6, n. II, p. I883, 2012.

MINISTÉRIO DA SAÚDE. Diretrizes para diagnóstico e tratamento da COVIDig (versão 04), maio, 2020. Disponível em: https://portaldeboaspraticas.iff.fiocruz.br/wpcontent/uploads/2020/o5/Sumario-Covidı-V4-8.04.pdf. Acesso em: 9 abr. 202I.

MITJÀ, O. et. al. A Cluster-Randomized Trial of Hydroxychloroquine for Prevention of Covid-19. The New England Journal of Medicine, 2020.

NGUYEN, A.A. et. al. Immunoglobulins in the treatment of covid-rg infection: Proceed with caution! Clinical immunology, v. 216, 2020.

NHS. Interleukin-6 inhibitors (tocilizumab or sarilumab) for hospitalised patients with COVID-i9 pneumonia (adults), 2021. Disponível em: https://www.cas.mhra.gov.uk/ViewandAcknowledgment/ViewAlert.aspx?AlertID=I03I44. Acesso em: 12 abr. 2021.

NIH. Antiviral drugs that are approved or under evaluation for the treatment of covid-ig, 202ra. Disponível em: https://www.covidigtreatmentguidelines.nih.gov/antiviral-therapy/. Acesso em: 30 mar. 202I.

NIH. The covid-I9 treatment guidelines panel's statement on the emergency use authorization of the bamlanivimab plus etesevimab combination for the treatment of covid-rig, 2021b. Disponível em: https://www.covidigtreatmentguidelines.nih.gov/statement-onbamlanivimab-plus-etesevimab-eua/. Acesso em: 30 mar. 2021.

NIH. What's new. COVID-i9 Treatment Guidelines, 202Ic. Disponível em: https://www.covidigtreatmentguidelines.nih.gov/whats-new/ Acesso em: 30 mar. 2021 .

PASIN, L. et al. Corticosteroids for patients with coronavirus disease 2019 (covid-19) with different disease severity: a meta-analysis of randomized clinical trials. Journal of Cardiothoracic and Vascular Anesthesia, v. 35, n. 2, p. 578-584, 2021.

PETER, W. et al. Tocilizumab in patients admitted to hospital with covid-I9 (recovery): preliminary results of a randomised, controlled, open-label, platform trial. Merdrxiv, 202I. Disponível em: https://www.medrxiv.org/content/IO.IIoI/202I.02.II.21249258vi.full.pdf. Acesso em: 12 abr. 2021. 
POYIADJI, N. et, al. Principle trial collaborative group. Azithromycin for community treatment of suspected COVID-I9 in people at increased risk of an adverse clinical course in the UK (PRINCIPLE): a randomised, controlled, open-label, adaptive platform trial. The Lancet, v. 397, p. 1063-1074, 2021.

RANG, H.P. Rang and dale farmacologia. Elsevier, v.8, 2016.

RECOVERY COLLABORATIVE GROUP. Azithromycin in patients admitted to hospital with covid-I9 (recovery): a randomised, controlled, open-label, platform trial. The Lancet, v. 397, p. 605-612, 2021a.

RECOVERY COLLABORATIVE GROUP. Dexamethasone in hospitalized patients with covid-I9. The New England Journal of Medicine, v. 384, n. 8, p. 693-704, 202rb.

RECOVERY COLLABORATIVE GROUP. Effect of hydroxychloroquine in hospitalized patients with covid-ı. New England Journal of Medicine, v. 383, n.21, p. 2030-40, 2020.

SALAMA, C, et al. Tocilizumab in Patients Hospitalized with Covid-I9 Pneumonia. New England Journal of Medicine. 2021;384:20-30.Epub January 7, 2021.

SCOTT, L.J. Tocilizumab: A review in rheumatoid arthritis. Drugs, v. 77, n. 17, p. 1865-1879, 2017.

SHAKOOR, H. et al. Immune-boosting role of vitamins D, C, E, zinc, selenium and omega-3 fatty acids: Could they help against COVID-I9? Maturitas, v.I43, p.I-9, 202I.

STONE, J.H. et al. Efficacy of Tocilizumab in Patients Hospitalized with Covid-i9 New England Journal of Medicine, v. 383, n. 24, p. 2333-2344, 2020.

REACT (THE WHO RAPID EVIDENCE APPRAISAL FOR COVID-I9 THERAPIES WORKING GROUP); Association Between Administration of Systemic Corticosteroids and Mortality Among Critically Ill Patients With COVID-19: A Meta-analysis. JAMA, v. 324, n. 13, p. 1330, 2020.

U.S. FOOD AND DRUG ADMINISTRATION (FDA). Disposições sobre a autorização de uso emergencial de plasma convalescente no tratamento de pacientes hospitalizados por COVID-I9, 202I. Disponível em https://www.fda.gov/media/I4I477/download. Acesso em: I2 abr. 2021.

VAN PAASSEN, J. et al. Corticosteroid use in covid-I9 patients: a systematic review and meta-analysis on clinical outcomes. Critical Care, v. 24, n. I, p. 696, 2020.

VARATHARAJ, A. \& GALEA, I. The blood-brain barrier in systemic inflammation. Brain, Behavior, And Immunity, [S.L.], v. 6o, p. I-12, 2017.

VEIGA, V.C. et al. Effect of tocilizumab on clinical outcomes at is days in patients with severe or critical coronavirus disease 2019: randomised controlled trial. BMJ, v.202I, p.372: n84, 2021. 
WANG, M. et al. Remdesivir and chloroquine effectively inhibit the recently emerged novel coronavirus (2019-ncov) in vitro. Cell Research, v. 30, n.3, p. 269-71, 2020.

WHO. Living guidance COVID-I9 Clinical management, 2021. Disponível em: https://www.who.int/publications/i/item/WHO-2019-nCoV-prophylaxes-202I-I. Acesso em: 30 mar. 2021. 